\title{
Mutation screening of germline TP53 mutations in high-risk Chinese breast cancer patients
}

\author{
Ava Kwong ${ }^{1,2,3^{*}}$ (D), Vivian Yvonne Shin ${ }^{1}$, Cecilia Y. S. Ho ${ }^{4}$, Chun Hang Au ${ }^{4}$, Thomas P. Slavin ${ }^{5,6}$, Jeffrey N. Weitzel, ${ }^{5,6}$, \\ Tsun-Leung Chan ${ }^{3,4}$ and Edmond S. K. Ma $\mathrm{Ma}^{3,4}$
}

\begin{abstract}
Background: Germline TP53 mutations are associated with Li-Fraumeni syndrome, a severe and rare hereditary cancer syndrome. Despite the rarity of germline TP53 mutations, the clinical implication for mutation carriers and their families is significant. The risk management of TP53 germline mutation carriers is more stringent than BRCA carriers, and radiotherapy should be avoided when possible.
\end{abstract}

Methods: TP53 gene mutation screening was performed in 2538 Chinese breast cancer patients who tested negative for BRCA mutations.

Results: Twenty TP53 mutations were identified with high next-generation sequencing concerning for germline mutations in Chinese breast cancer families. The majorities of the TP53 carriers had early-onset, hormone receptorpositive breast cancer, and had strong family history of cancer. Among all, 11 patients carried a germline mutation and 6 of which were likely de novo germline mutations. In addition, 1 case was suspected to be induced by chemotherapy or radiation, as this patient had no significant family history of cancer and aberrant clonal expansion can commonly include TP53 mutations. Furthermore, we have identified one mosaic LFS case. Two novel mutations (c.524_547dup and c.529_546del) were identified in patients with early-onset.

Conclusions: In view of the high lifetime risk of malignancy, identification of patients with germline TP53 mutations are important for clinicians to aid in accurate risk assessment and offer surveillance for patients and their families.

Keywords: Hereditary breast cancer, TP53 mutation, Chinese, Breast cancer risk

\section{Background}

Li-Fraumeni syndrome (LFS) is a rare autosomal genetic disorder which is frequently associated with germline TP53 mutations. Germline TP53 mutations are seen in $70 \%$ of families with LFS features. Individual with the mutation commonly present with LFS spectrum tumors (sarcoma, brain tumor, adrenocortical carcinoma, leukemia,

\footnotetext{
* Correspondence: akwong@asiabreastregistry.com

'Department of Surgery, The University of Hong Kong and University of Hong Kong-Shenzhen Hospital, Hong Kong SAR, China

${ }^{2}$ Department of Surgery, Hong Kong Sanatorium \& Hospital, Hong Kong SAR, China

Full list of author information is available at the end of the article
}

germ cell tumor and breast cancer) $[1,2]$. The lifetime risk of breast cancer in TP53 mutation carriers is up to $80-90 \%$, which is even higher than those harboring a $B R C A 1$ or $B R C A 2$ (BRCA) mutation: the most commonly identified high penetrance germline gene mutations in hereditary breast cancer [3].

Although rare, germline TP53 mutations are estimated to occur in up to $1 \%$ of all breast cancer cases [4]. Very early-onset of breast cancer is a common characteristic of TP53 mutation carriers in which the median age being 27-30 years old [5]. TP53 breast tumors are usually enriched with HER2-positive receptors, and $84 \%$ are either

(c) The Author(s). 2020 Open Access This article is licensed under a Creative Commons Attribution 4.0 International License, which permits use, sharing, adaptation, distribution and reproduction in any medium or format, as long as you give appropriate credit to the original author(s) and the source, provide a link to the Creative Commons licence, and indicate if changes were made. The images or other third party material in this article are included in the article's Creative Commons licence, unless indicated otherwise in a credit line to the material. If material is not included in the article's Creative Commons licence and your intended use is not permitted by statutory regulation or exceeds the permitted use, you will need to obtain permission directly from the copyright holder. To view a copy of this licence, visit http://creativecommons.org/licenses/by/4.0/ The Creative Commons Public Domain Dedication waiver (http://creativecommons.org/publicdomain/zero/1.0/) applies to the data made available in this article, unless otherwise stated in a credit line to the data. 
estrogen and/or progesterone receptor positive [6, 7]. Patients with TP53 mutations have also been shown to have a shorter survival when compared to non-carriers [8].

The National Comprehensive Cancer Network (NCCN) has published testing and management guidelines for TP53 gene mutation carriers. Under the NCCN guidelines, TP53 mutation testing is recommended for early-onset breast cancer patients (age of diagnosis $<31$ ) or those who meet classic LFS or Chompret criteria. It is also recommended that women who are TP53 mutations carriers have breast surveillance similar to that of $B R C A$ mutation carriers, and in addition, receive an annual total body MRI scan and skin cancer screening, and colonoscopy every $2-5$ years beginning at 25 years of age. Although there is still no actionable drug which can target TP53 mutations with good clinical trial data support, evidence shows that patients who carried TP53 mutation are less responsive to low dose radiation and have a higher risk of new malignancies induced by radiotherapy [9]. Hence, TP53 mutation status does have its importance in clinical management.

To date, over 250 types of TP53 mutations are included in the IARC TP53 Database and over $70 \%$ of them are missense mutations (http://p53.iarc.fr/). Missense mutations are often more challenging to classify and interpret than loss of function mutations. Therefore, obtaining more phenotypic data on unique missense mutations is important for the literature. Furthermore, germline TP53 literature has historically been confounded by aberrant clonal expansion (ACE) [10], an entity that describes low level hematopoietic progenitor cell mutations, usually in leukemia related genes confined to the blood compartment [11]. ACE has been shown to associate with advancing age and clinical exposures like chemotherapy and radiation. ACE can be a risk factor for leukemia, atherosclerotic vascular disease, and associated with increased all-cause mortality [11].

In a cohort of 240 Chinese women with early-onset of breast cancer (age $<35$ ) or with first- or second-degree relatives with breast and/or ovarian cancer, TP53 mutation carriers were found in $1 \%$ of the cohort [12]. However, in Canada, there were no pathogenic mutations identified in a cohort of 95 women with early-onset of breast cancer $($ age $<30)$ [13]. This suggests that the clinical characteristics of TP53 carriers vary across different ethnicities and countries. The frequency of TP53 mutations also remains largely unknown in the Chinese population, therefore, our study aims to investigate the prevalence of TP53 mutation in Chinese breast cancer patients and unravel the clinical characteristics of TP53 mutations in their families.

\section{Methods}

\section{Participants and selection criteria}

TP53 gene mutation screening was performed on 2538 Chinese breast cancer patients with no BRCA1 and
BRCA2 germline mutations. Patients were recruited from the Hong Kong Hereditary and High Risk Breast Cancer Program (HRBCP) through the Hong Kong Hereditary Breast Cancer Family Registry from March 2007 to August 2019. Patient selection criteria was as follows: (1) patients had at least one first- or second- degree relative with breast and/or ovarian cancer, regardless of age; (2) the age at breast cancer diagnosis was under 45 years; (3) patients with bilateral breast cancer; (4) patients with triple-negative hormone receptors breast cancers, (5) cancers with medullary type histology; (6) patients having at least one relative with $B R C A$-associated cancer other than breast and ovarian cancer (such as stomach or prostate cancers) or known to be BRCA mutation related family; (7) patients with male breast cancer.

\section{DNA extraction}

Blood, hair follicles and/or buccal swab DNA samples were collected from patients. Genomic DNA extraction was performed using QIAamp DNA Blood Mini Kit (Qiagen, Hilden, Germany) or QIAsymphony DNA Mini Kit (Qiagen) according to manufacturer's instructions. Genomic DNA was quantified using a Qubit dsDNA BR Assay Kit and a Qubit 2.0 Fluorometer (Life Technologies, USA).

\section{Sequencing of TP53 gene}

Extracted DNA was applied to the QIAseq Human BRCA1 and BRCA2 Plus Panel DHS-103Z (Qiagen). Sequencing libraries were prepared according the QIAseq ${ }^{\text {tw }}$ Targeted DNA Panel protocol (Qiagen). The libraries were pooled and sequenced on MiSeq or NextSeq (Illumina, San Diego, CA) instruments to reach minimum sequencing depth of 50-fold. Median coverage typically ranged between 200-300X.

To confirm germline mutations, Sanger sequencing of specific mutations was carried out on blood, hair follicles, and/or buccal swab DNA.

\section{Bioinformatics analysis}

The bioinformatics analysis was performed on a Cray XC30 supercomputer (Cray, Seattle, WA). Paired sequencing reads were mapped to the human reference genome sequence GRCh37/hg19 using BWA-MEM v0.7.7 by default parameters [14]. Post-alignment primer clipping and unique molecular identifier (UMI) extraction were performed using BAMClipper [15]. Samples having at least $75 \%$ gene-specific primers with at least 100 detected UMI per primer were considered to pass quality control and subject to variant calling by FreeBayes v1.0.2-15 [16]. Called variants with variant allelic fraction (VAF) of at least 5\% were annotated by Ensembl Variant Effect Predictor v75 [17]. Variants with minor allele frequency of at least 1\% reported by The 1000 
Genomes Projects [18] were excluded from manual variant curation. Variants in exon and at least $10 \mathrm{bp}$ of the flanking introns were reported and described according to the standardized recommendations of the Human Genome Variation Society (HGVS) nomenclature [19]. Variant descriptions were checked by IARC TP53 database (http://p53.iarc.fr/) and Mutalyzer Name Checker (http://mutalyzer.nl). Variants in this study were interpreted based on classification from ClinVar database (https://www.ncbi.nlm.nih.gov/clinvar/) with clinical adjustment with reference to the classic for Li-Fraumeni syndrome criteria [2].

\section{Molecular analysis of de novo germline mutations}

Mutations from families in which both parents tested negative were presumed as de novo mutations. Haplotype analysis was also performed to confirm de novo cases. In cases where the patient had no first or seconddegree relatives with cancer history or positive test result, and the blood samples from the patient's parent were unavailable, the patient was considered as likely de novo.

\section{Statistical analysis}

Fisher's exact test and Wilcoxon rank sum test were used to study the relationship between clinicopathological variables and mutation status. The limit of significance for all analyses was defined as $P$-value of $<0.05$. Data analyses were performed using statistical software $\mathrm{R}$ (version 3.4.2) [20].

\section{Results}

In a cohort of 2538 breast cancer patients, there were 28 PALB2 and 2 PTEN mutation identified, which were excluded from the study. Among 2508 patients, the mean age at diagnosis was 45.63 years (range 18-95). Of all primary tumors, $1760(75.41 \%)$ were hormone receptor positive, 211 (9.04\%) were HER2+, and 327 (14.01\%) were triple-negative. A positive family history of breast cancer (first- or second-degree relatives) was seen among $922(36.76 \%)$ of the patients and 473 (18.86\%) of the patients had a family history with $\geq 3$ different types of cancers in their first- and second-degree relatives.

TP53 mutations were infrequent in this cohort. Only 20 different mutations $(0.80 \%)$ were identified among the 2508 breast cancer patients. The mean age at diagnosis of breast cancer for the mutation carriers and noncarriers were 31.65 years and 45.74 years ( $p$-value < 0.001), respectively. In TP53 mutation carriers, the majority of the tumors were hormone receptor-positive (16/21, 76.19\%) (OR compare with non-carriers: 1.04, 95\% CI: $0.363-3.661 ; p$-value $=1)$. A positive family history of breast cancer (among first- and second- degree relatives) was reported in 5 (25\%) TP53 mutation carriers compared to 917 (36.86\%) non-carriers (OR 0.571, 95\% CI: $0.162-1.660, p$-value $=0.355)$. Moreover, there were $8(40 \%)$ mutation carriers with a family history of $\geq 3$ different types of cancers in first- and seconddegree relatives compared to 465 (18.69\%) of noncarriers (OR: 2.90, 95\% CI 1.022-7.764, p-value =0.038). Characteristics of mutation carriers and non-carriers are shown in Table 1.

The majority of the mutations (15/20) identified were missense mutations, followed by 2 nonsense mutations, 2 deletions/insertions and 1 splice site mutation (Tables 2 and 3). By testing ancillary materials, multiple germ layers and/or clinical data to interrogate germline status on the 20 carriers, 11 (55\%) patients were confirmed to carry a germline mutation, $2(10 \%)$ patients were confirmed to have de novo germline mutations (Fig. 1), and $4(20 \%)$ were presumed to have de novo germline mutations based on the negative test result of TP53 mutation among multiple family members and/or lack of cancer history in families. Five of the patients were deceased and three of the patients refused further investigation on family studies, some information were no longer traceable. In all, 70\% had early-onset of breast cancer $(<35$ years) and $60 \%$ had bilateral breast cancer. Interestingly, we found that $25 \%(5 / 20)$ of the patients had no family history of cancer, 2 patients had bilateral breast cancer, one had bilateral breast cancer and thyroid cancer and one had multiple cancers in breast and brain.

Novel mutations (c.524_547dup and c.529_546del) were seen in 2 patients with both diagnosed breast cancer at age below 40. One of the novel mutation carriers (F19) shows equivocal result in both of her blood, hair follicle and buccal swab DNA with trace amount of TP53 duplication, serving as evidence of mosaicism or ACE (in Table 3). The other novel TP53 carrier (F06) was de novo germline mutation, with significance family history of cancer.

In this cohort, there were 5 suspected germline mutation cases (F03, F05, F08, F11 and F18); these patients had strong family history with cancers and fulfil either Li-Fraumeni-like (LFL) criteria and/or Chompret criteria, however, family cascade testing was not possible due to loss of follow-up or family members refusing testing. In addition, we suspected that one of the cases (F20) was likely ACE induced by chemotherapy or radiation (Table 3); this patient had breast cancers at age $>45$, received chemotherapy before the genetic test, and had no other significant personal or family history of cancer and hence is likely not a germline related.

\section{Discussion}

Among 2508 Chinese breast cancer patients, we identified 18 germline TP53 and 2 ACE/mosaic TP53 cases. Of 18 germline cases, two of them did not meet the 
Table 1 Characteristics of Chinese breast cancer patients screened for TP53 mutations

\begin{tabular}{|c|c|c|c|c|c|c|c|}
\hline & $\begin{array}{l}\text { Mutation Negative } \\
N=2488\end{array}$ & $\%$ & $\begin{array}{l}T P 53+ \\
N=20\end{array}$ & $\%$ & $\begin{array}{l}\text { Total } \\
N=2508\end{array}$ & $\%$ & $\begin{array}{l}\text { P-value } \\
\text { (Wilcoxon rank sum test/ } \\
\text { Fisher Exact Test) }\end{array}$ \\
\hline Mean/Median age at Diagnosis & $45.74 / 44$ & & $31.65 / 30$ & & $45.63 / 44$ & & $<0.001$ \\
\hline Age range & $18-95$ & & $18-47$ & & 18-95 & & \\
\hline Bilateral cases & 439 & $17.64 \%$ & 12 & $60.00 \%$ & 451 & $17.98 \%$ & $<0.001$ \\
\hline \multicolumn{8}{|l|}{ Age at breast cancer diagnosis } \\
\hline$\leq \mathbf{2 9}$ & 116 & $4.66 \%$ & 9 & $45.00 \%$ & 125 & $4.98 \%$ & $<0.001$ \\
\hline $30-39$ & 637 & $25.60 \%$ & 6 & $30.00 \%$ & 643 & $25.64 \%$ & \\
\hline $40-49$ & 998 & $40.11 \%$ & 5 & $25.00 \%$ & 1003 & $39.99 \%$ & \\
\hline$\geq \mathbf{5 0}$ & 737 & $29.62 \%$ & 0 & $0.00 \%$ & 737 & $29.39 \%$ & \\
\hline \multicolumn{8}{|c|}{ Family history of breast cancer (in first and second degree relatives) } \\
\hline Yes & 917 & $36.86 \%$ & 5 & $25.00 \%$ & 922 & $36.76 \%$ & 0.355 \\
\hline No & 1571 & $63.14 \%$ & 15 & $75.00 \%$ & 1586 & $63.24 \%$ & \\
\hline \multicolumn{8}{|c|}{ Family history of $>=3$ different types of cancers (in first and second degree relatives) } \\
\hline Yes & 465 & $18.69 \%$ & 8 & $40.00 \%$ & 473 & $18.86 \%$ & 0.038 \\
\hline No & 2023 & $81.31 \%$ & 12 & $60.00 \%$ & 2035 & $81.14 \%$ & \\
\hline \multicolumn{8}{|l|}{ Histology ${ }^{a}$} \\
\hline & $N=2927$ & & $N=32$ & & $N=2959$ & & \\
\hline Ductal & 1991 & $71.21 \%$ & 17 & $58.62 \%$ & 2008 & $71.08 \%$ & 0.283 \\
\hline Lobular & 94 & $3.36 \%$ & 1 & $3.45 \%$ & 95 & $3.36 \%$ & \\
\hline DCIS & 498 & $17.81 \%$ & 9 & $31.03 \%$ & 507 & $17.95 \%$ & \\
\hline Others & 213 & $7.62 \%$ & 2 & $6.90 \%$ & 215 & $7.61 \%$ & \\
\hline Unclassified & 131 & - & 3 & - & 134 & - & \\
\hline \multicolumn{8}{|c|}{ Molecular subtypes ${ }^{\mathrm{a}}$ (excluded in-situ CA) } \\
\hline & $N=2429$ & & $N=23$ & & $N=2452$ & & \\
\hline Hormone receptor + & 1744 & $75.40 \%$ & 16 & $76.19 \%$ & 1760 & $75.41 \%$ & 0.291 \\
\hline Hormone receptor - & 36 & $1.56 \%$ & 0 & $0.00 \%$ & 36 & $1.54 \%$ & \\
\hline HER2+ & 207 & $8.95 \%$ & 4 & $19.05 \%$ & 211 & $9.04 \%$ & \\
\hline TNBC & 326 & $14.09 \%$ & 1 & $4.76 \%$ & 327 & $14.01 \%$ & \\
\hline Unclassified & 116 & - & 2 & - & 118 & - & \\
\hline
\end{tabular}

Abbreviation: DCIS ductal carcinoma in situ, HER2 human epidermal growth factor receptor 2, TNBC Triple-negative breast cancer

${ }^{\text {a } C o u n t ~ f o r ~ e a c h ~ p r i m a r y ~ o f ~ b i l a t e r a l ~ c a s e s ~}$

NCCN guidelines for TP53 genetic testing but the families had LFL syndrome (Table 2). In general, germline TP53 families had at least one member with LFS tumor spectrum i.e. sarcoma, brain tumor, breast cancer, leukemia, bronchoalveolar lung carcinomas, germ cell tumor or adrenocortical carcinoma [1, 2, 21-23]. However, we found that $16.67 \%$ ( 3 of 18 ) of the patients had no family history of cancer.

Among 374 patients in our study with early-onset breast cancer (age $<35$ ), the detection rate of a TP53 germline mutation was $3.74 \%$ which is comparable to other studies in the West $(2-7.1 \%)[24,25]$, and among Chinese high risk breast patients $(1-5 \%)[12,26,27]$ (Table 2). A study of French-Canadian cancer families suggested that women with breast cancer before the age of 50 with no family history of cancer still warrant screening for TP53 mutations, even though the mutation frequency $(0.5 \%)$ is low compare to $B R C A$ mutations (4.8\%) [28].

Interestingly, there were 6 (33.3\%) de novo or likely de novo cases. Another study on early-onset cancer study suggests that the frequency of de novo TP53 mutations is $7-20 \%$ [29]. There were two TP53 mutations, c.490A > G (F04) and c.536A > G (F07) both of the families showed characteristics of classical LFS. Their families have significant family history of sarcoma, although ClinVar has classified them as variant of unknown significance (VUS), we believe that the pathogenicity of these two variants should be further determined based on their family histories. With a significant family 


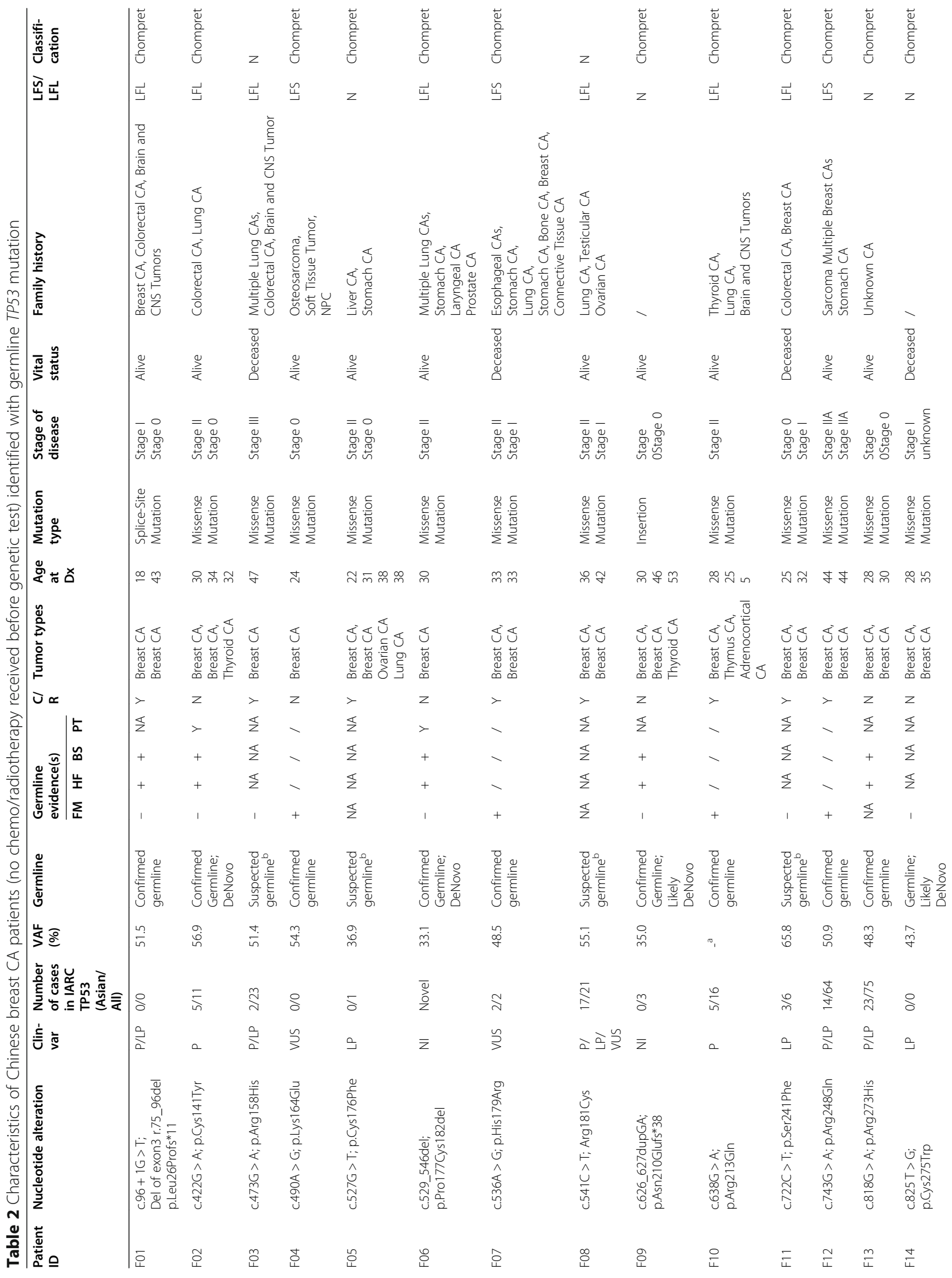


Kwong et al. BMC Cancer ～(2020) 20:1053

Page 6 of 10

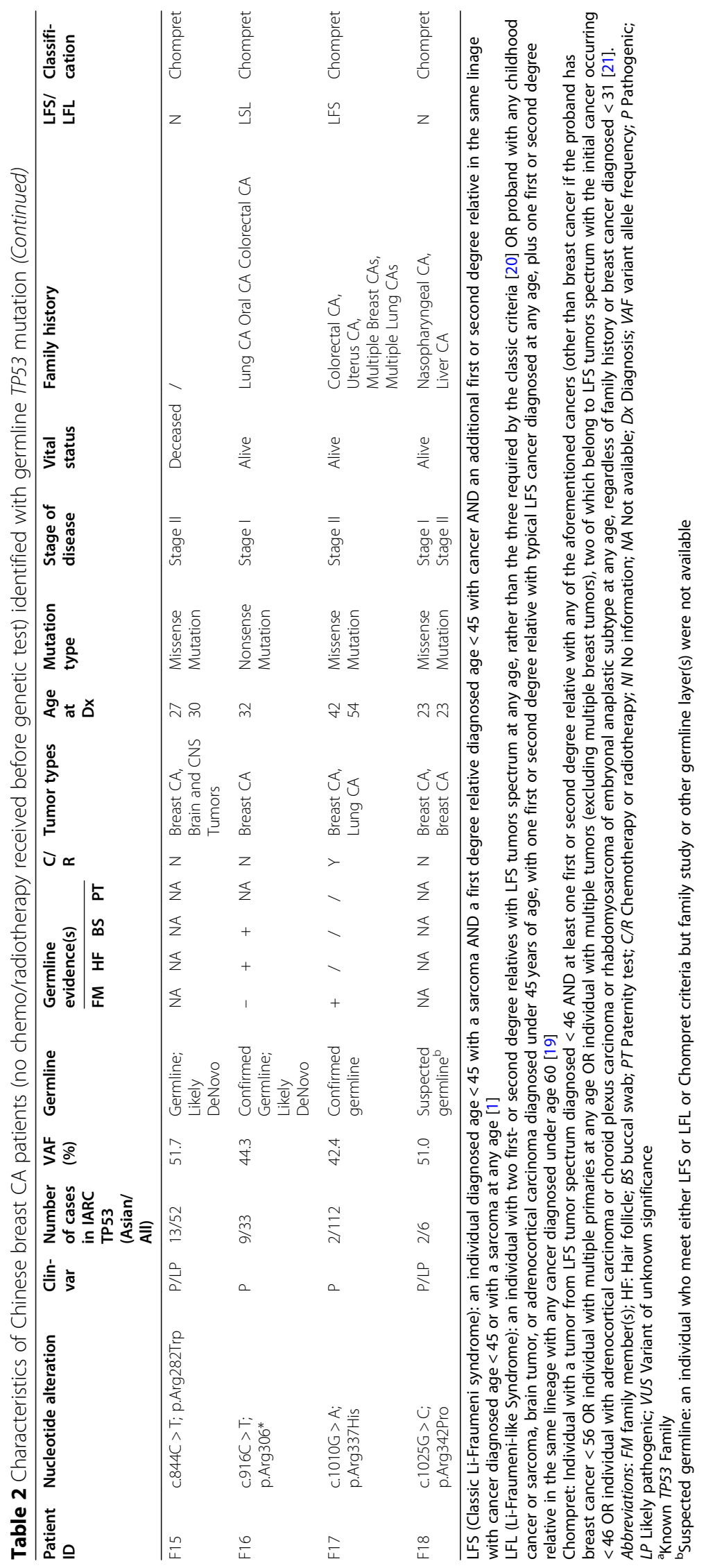




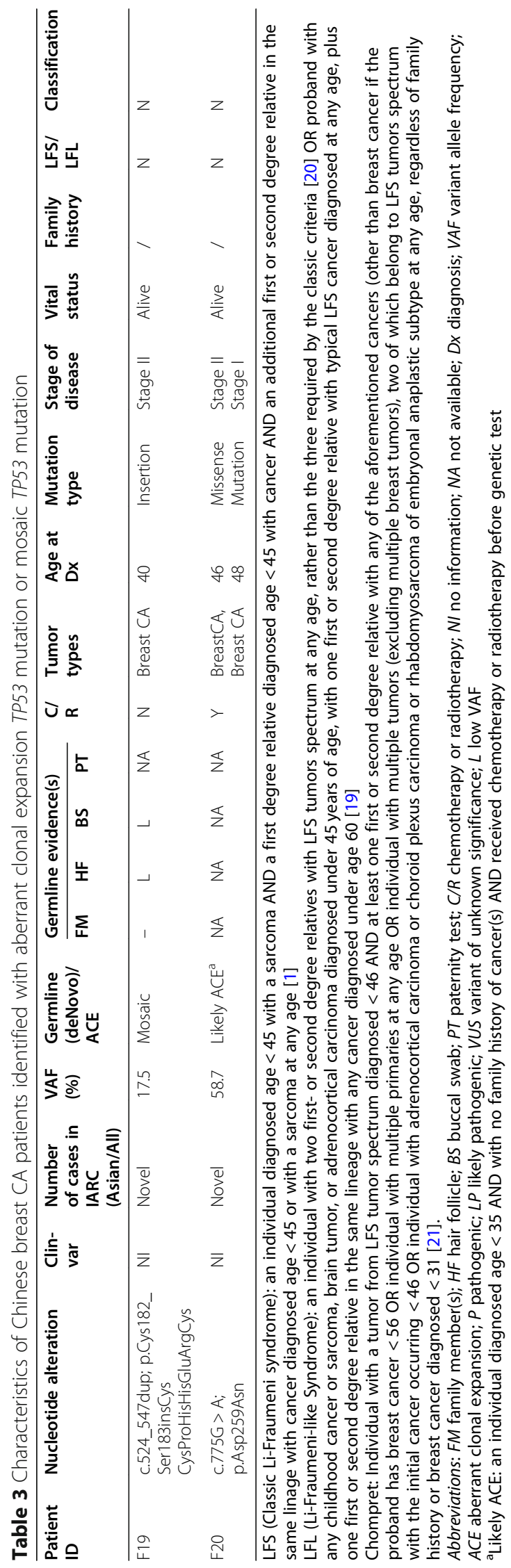




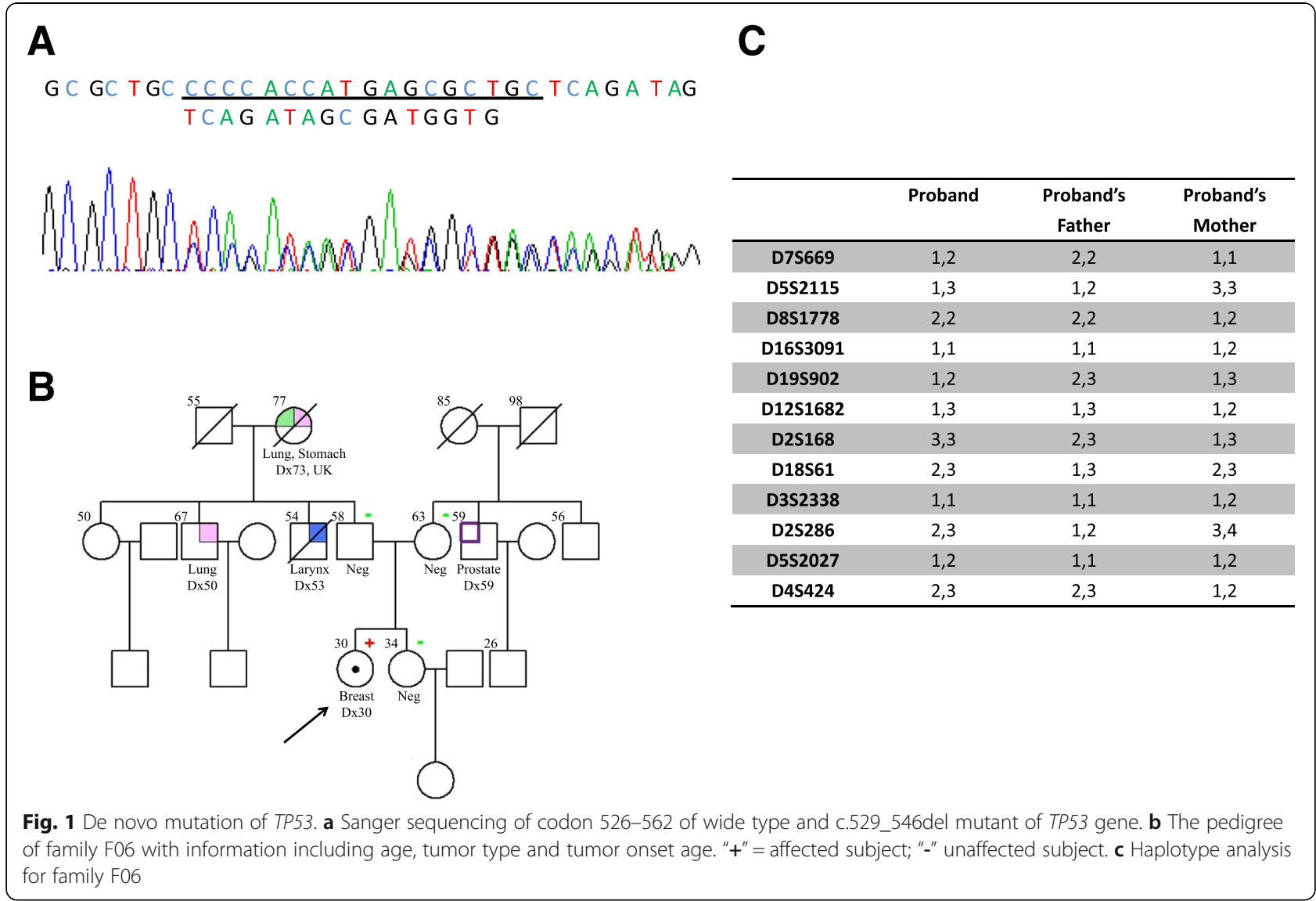

history of cancers, these families have been offered high risk surveillance.

The mutation TP53, c.1010G > A, has been previously reported as founder mutation in Southern Brazilian [30]. Interestingly, it was detected in one of the Chinese families who had breast and lung cancer and multiple family cancers. The mutation TP53, c.529_546del, has been identified somatically in thyroid cancer [31], small cell lung cancer [32] and breast cancer [24, 33]. We detected this mutation in one of the families who had breast cancer at age 30 and a family history of multiple cancers. The variant allele fraction (VAF) was at 33\% by NGS, which was lower than the average range of TP53 VAFs identified in our study. Further analysis was performed on a buccal swab by Sanger sequencing in which the VAF was 50\% and therefore the mutation was confirmed to be germline.

In another family (F19), we detected a $17.5 \%$ VAF by NGS, which was much lower than the normal germline range of $40-60 \%$ VAF. Further analysis on hair follicles and buccal swab by both NGS and Sanger sequencing showed trace amounts of the mutation, therefore testing on tumor tissue would be able to confirm somatic mosaicism or ACE [10], however, the patient received neoadjuvant chemotherapy before surgery and there was no tissue available for further testing.
Radiation induced genomic instability causing aberrant hematopoietic stem/progenitor cells mobilized into the peripheral blood circulation result in ACE sometimes involving TP53 [10, 34]. In one of the patients who was only tested after chemotherapy has been administered, breast cancer was diagnosed at old age $(>35)$ and there was no cancer history in their families, suggesting the variant was more likely due to ACE rather than LFS.

Increased risk of secondary malignancies in TP53 mutation carriers with radiation exposure has been reported [35]. In a preclinical study of 6 germline TP53 mutated breast cancer patients who received adjuvant radiotherapy, 3 later developed ipsilateral breast recurrences, 4 developed contralateral breast cancers, 2 developed radiotherapyinduced cancers, and 2 developed new primaries (1 of which was an ipsilateral chest wall angiosarcoma and the other was a grade 2 ethmoidal leiomyosarcoma) [36].

\section{Conclusion}

Overall, our study shows the spectrum of TP53 germline mutations in a Chinese cohort and also clinical characteristics of Chinese TP53 carriers and their families which may help clinicians identify patients for TP53 mutation screening. Young aged (even without a cancer family history) women with breast cancer is a major 
association and should be considered for TP53 genetic testing. Identification of a TP53 mutation may also affect the treatment options for these patients, i.e. potentially minimizing the use of radiation to prevent radiationrelated malignancies [37]. Moreover, our findings may aid in the development of new guidelines for TP53 screening in breast cancer patients with Chinese ethnicity.

\section{Supplementary information}

Supplementary information accompanies this paper at https://doi.org/10 1186/s12885-020-07476-y.

\section{Additional file 1}

\section{Abbreviations}

ACE: Aberrant clonal expansion; HRBCP: Hong Kong Hereditary and High Risk Breast Cancer Program; HGVS: Human Genome Variation Society; LFS: LiFraumeni syndrome; NCCN: The National Comprehensive Cancer Network; UMI: Unique molecular identifier; VAF: Variant allelic fraction

\section{Acknowledgements}

We thank Wing Pan Luk and Ling Hiu Fung from the Hong Kong Sanatorium \& Hospital for assistance with processing sequencing data and statistical analyses. We would also like to thank doctors and nurses from the Department of Surgery, the University of Hong Kong, and members of the Hong Kong Breast cancer research groups from the Departments of Surgery and Oncology of contributing Hospitals for recruitment of patients.

\section{Authors' contributions}

The study was designed by AK, EM, JW, TC and TS. CH and CA performed the experiments. CA performed the bioinformatics analysis and interpreted the results. CH and VS collected data and drafted the manuscript. AK, EM, JW, TC and TS reviewed the manuscript. All authors read and approved the final manuscript

\section{Funding}

Dr. Ellen Li Charitable Foundation; Kerry Kuok Foundation; Health and Medical Research Fund (03143406); Seed Fund for Basic Research (201611159186 and 104005409), Asian Fund for Cancer Research and Hong Kong Hereditary Breast Cancer Family Registry; National Cancer Institute (NCl) of the National Institutes of Health $(\mathrm{NIH})$, award numbers R01CA242218 (PI: J. Weitzel). The content is solely the responsibility of the authors and does not necessarily represent the official views of the $\mathrm{NIH}$.

\section{Availability of data and materials}

The data that support the findings of this study are available from the corresponding author upon reasonable request.

\section{Ethics approval and consent to participate}

This study was approved by the Institutional Review Board of the University of Hong Kong/Hospital Authority West Cluster and other contributing hospitals in Hong Kong (UW06-274 T1299) and was conducted in accordance with the Declaration of Helsinki. All recruited participants provided written informed consent for sample and data collection in this study.

\section{Consent for publication}

Not applicable.

\section{Competing interests}

The authors declare that they have no conflicts of interest.

\section{Author details}

'Department of Surgery, The University of Hong Kong and University of Hong Kong-Shenzhen Hospital, Hong Kong SAR, China. ${ }^{2}$ Department of Surgery, Hong Kong Sanatorium \& Hospital, Hong Kong SAR, China. ${ }^{3}$ Hong
Kong Hereditary Breast Cancer Family Registry, Hong Kong SAR, China. ${ }^{4}$ Department of Pathology, Division of Molecular Pathology, Hong Kong Sanatorium \& Hospital, Hong Kong SAR, China. ${ }^{5}$ Department of Medical Oncology \& Therapeutics Research, Division of Clinical Cancer Genetics, City of Hope, Duarte, CA, USA. ${ }^{6}$ Department of Population Sciences, Beckman Research Institute of City of Hope, Duarte, CA, USA.

Received: 27 May 2020 Accepted: 30 September 2020

Published online: 02 November 2020

\section{References}

1. Hartley AL, Birch JM, Kelsey AM, Marsden HB, Harris M, Teare MD. Are germ cell tumors part of the Li-Fraumeni cancer family syndrome? Cancer Genet Cytogenet. 1989;42(2):221-6.

2. Li FP, Fraumeni JF Jr, Mulvihill JJ, Blattner WA, Dreyfus MG, Tucker MA, Miller RW. A cancer family syndrome in twenty-four kindreds. Cancer Res. 1988; 48(18):5358-62.

3. Lalloo F, Evans DG. Familial breast cancer. Clin Genet. 2012;82(2):105-14.

4. Malkin D, Li FP, Strong LC, Fraumeni JF Jr, Nelson CE, Kim DH, Kassel J, Gryka MA, Bischoff FZ, Tainsky MA, et al. Germ line p53 mutations in a familial syndrome of breast cancer, sarcomas, and other neoplasms. Science. 1990;250(4985):1233-8.

5. Apostolou P, Fostira F. Hereditary breast cancer: the era of new susceptibility genes. Biomed Res Int. 2013;2013:747318.

6. Wilson JR, Bateman AC, Hanson H, An Q, Evans G, Rahman N, Jones JL, Eccles DM. A novel HER2-positive breast cancer phenotype arising from germline TP53 mutations. J Med Genet. 2010;47(11):771-4.

7. Masciari S, Dillon DA, Rath M, Robson M, Weitzel JN, Balmana J, Gruber SB, Ford JM, Euhus D, Lebensohn A, et al. Breast cancer phenotype in women with TP53 germline mutations: a Li-Fraumeni syndrome consortium effort. Breast Cancer Res Treat. 2012;133(3):1125-30.

8. Olivier M, Langerod A, Carrieri P, Bergh J, Klaar S, Eyfjord J, Theillet C, Rodriguez C, Lidereau R, Bieche I, et al. The clinical value of somatic TP53 gene mutations in 1,794 patients with breast cancer. Clin Cancer Res. 2006; 12(4):1157-67.

9. Evans DG, Birch JM, Ramsden RT, Sharif S, Baser ME. Malignant transformation and new primary tumours after therapeutic radiation for benign disease: substantial risks in certain tumour prone syndromes. J Med Genet. 2006:43(4):289-94.

10. Weitzel JN, Chao EC, Nehoray B, Van Tongeren LR, LaDuca H, Blazer KR, Slavin T, Facmg D, Pesaran T, Rybak C, et al. Somatic TP53 variants frequently confound germ-line testing results. Genet Med. 2018;20(8):809-16.

11. Jaiswal S, Fontanillas P, Flannick J, Manning A, Grauman PV, Mar BG, Lindsley RC, Mermel CH, Burtt N, Chavez A, et al. Age-related clonal hematopoiesis associated with adverse outcomes. N Engl J Med. 2014; 371(26):2488-98.

12. Cao AY, Jin W, Shi PC, Di GH, Shen ZZ, Shao ZM. Identification and characterization of two novel germ line p53 mutations in the non-LFS/nonLFL breast cancer families in Chinese population. Breast Cancer Res Treat. 2010;119(2):295-303.

13. Ginsburg OM, Akbari MR, Aziz Z, Young R, Lynch $H$, Ghadirian P, Robidoux A, Londono J, Vasquez G, Gomes M, et al. The prevalence of germ-line TP53 mutations in women diagnosed with breast cancer before age 30. Familial Cancer. 2009:8(4):563-7.

14. Li H: Aligning sequence reads, clone sequences and assembly contigs with BWA-MEM. arXiv:13033997v2 2013.

15. Au CH, Ho DN, Kwong A, Chan TL, Ma ESK. BAMClipper: removing primers from alignments to minimize false-negative mutations in amplicon nextgeneration sequencing. Sci Rep. 2017;7(1):1567.

16. Garrison E, Marth G: Haplotype-based variant detection from short-read sequencing. arXiv:12073907 2012

17. McLaren W, Gil L, Hunt SE, Riat HS, Ritchie GR, Thormann A, Flicek P, Cunningham F. The Ensembl variant effect predictor. Genome Biol. 2016; 17(1):122.

18. Genomes Project C, Auton A, Brooks LD, Durbin RM, Garrison EP, Kang HM, Korbel JO, Marchini JL, McCarthy S, McVean GA, et al. A global reference for human genetic variation. Nature. 2015:526(7571):68-74.

19. den Dunnen JT, Dalgleish R, Maglott DR, et al. HGVS recommendations for the description of sequence variants: 2016 update. Hum Mutat. 2016:37(6): 564-9. 
20. R Core Team. R: a language and environment for statistical computing. Vienna: R Foundation for statistical computing; 2019. URL https://www.Rproject.org/.

21. Birch JM, Hartley AL, Tricker KJ, Prosser J, Condie A, Kelsey AM, Harris M, Jones $\mathrm{PH}$, Binchy $\mathrm{A}$, Crowther $\mathrm{D}$, et al. Prevalence and diversity of constitutional mutations in the p53 gene among 21 Li-Fraumeni families. Cancer Res. 1994;54(5):1298-304.

22. Eeles RA. Germline mutations in the TP53 gene. Cancer Surv. 1995;25:101-24.

23. Chompret A, Abel A, Stoppa-Lyonnet D, Brugieres L, Pages S, Feunteun J, Bonaiti-Pellie C. Sensitivity and predictive value of criteria for p53 germline mutation screening. J Med Genet. 2001;38(1):43-7.

24. Boyault S, Drouet Y, Navarro C, Bachelot T, Lasset C, Treilleux I, Tabone E, Puisieux A, Wang Q. Mutational characterization of individual breast tumors: TP53 and PI3K pathway genes are frequently and distinctively mutated in different subtypes. Breast Cancer Res Treat. 2012;132(1):29-39.

25. Carraro DM, Koike Folgueira MA, Garcia Lisboa BC, Ribeiro Olivieri EH, Vitorino Krepischi AC, de Carvalho AF, de Carvalho Mota LD, Puga RD, do Socorro Maciel M, Michelli RA, et al. Comprehensive analysis of BRCA1, BRCA2 and TP53 germline mutation and tumor characterization: a portrait of early-onset breast cancer in Brazil. PLoS One. 2013;8(3):e57581.

26. Lee DS, Yoon SY, Looi LM, Kang P, Kang IN, Sivanandan K, Ariffin H, Thong MK, Chin KF, Mohd Taib NA, et al. Comparable frequency of BRCA1, BRCA2 and TP53 germline mutations in a multi-ethnic Asian cohort suggests TP53 screening should be offered together with BRCA1/2 screening to earlyonset breast cancer patients. Breast Cancer Res. 2012;14(2):R66.

27. Yang X, Wu J, Lu J, Liu G, Di G, Chen C, Hou Y, Sun M, Yang W, Xu X, et al. Identification of a comprehensive spectrum of genetic factors for hereditary breast cancer in a Chinese population by next-generation sequencing. PLoS One. 2015;10(4):e0125571.

28. Arcand SL, Akbari MR, Mes-Masson AM, Provencher D, Foulkes WD, Narod SA, Tonin PN. Germline TP53 mutational spectrum in French Canadians with breast cancer. BMC Med Genet. 2015;16:24.

29. Gonzalez KD, Buzin CH, Noltner KA, Gu D, Li W, Malkin D, Sommer SS. High frequency of de novo mutations in Li-Fraumeni syndrome. J Med Genet. 2009;46(10):689-93.

30. Paskulin DD, Giacomazzi J, Achatz MI, Costa S, Reis RM, Hainaut P, dos Santos SE, Ashton-Prolla P. Ancestry of the Brazilian TP53 c.1010G>A (p. Arg337His, R337H) Founder Mutation: Clues from Haplotyping of Short Tandem Repeats on Chromosome 17p. PLoS One. 2015;10(11):e0143262.

31. Landa I, Ibrahimpasic T, Boucai L, Sinha R, Knauf JA, Shah RH, Dogan S, Ricarte-Filho JC, Krishnamoorthy GP, Xu B, et al. Genomic and transcriptomic hallmarks of poorly differentiated and anaplastic thyroid cancers. J Clin Invest. 2016;126(3):1052-66.

32. Iwakawa R, Kohno T, Totoki Y, Shibata T, Tsuchihara K, Mimaki S, Tsuta K, Narita Y, Nishikawa R, Noguchi M, et al. Expression and clinical significance of genes frequently mutated in small cell lung cancers defined by whole exome/RNA sequencing. Carcinogenesis. 2015;36(6):616-21.

33. Silwal-Pandit L, Vollan HK, Chin SF, Rueda OM, McKinney S, Osako T, Quigley DA, Kristensen VN, Aparicio S, Borresen-Dale AL, et al. TP53 mutation spectrum in breast cancer is subtype specific and has distinct prognostic relevance. Clin Cancer Res. 2014;20(13):3569-80.

34. Schuler N, Palm J, Schmitz S, Lorat Y, Rube CE. Increasing genomic instability during cancer therapy in a patient with Li-Fraumeni syndrome. Clin Transl Radiat Oncol. 2017;7:71-8.

35. Kony SJ, de Vathaire F, Chompret A, Shamsaldim A, Grimaud E, Raquin MA, Oberlin O, Brugieres L, Feunteun J, Eschwege F, et al. Radiation and genetic factors in the risk of second malignant neoplasms after a first cancer in childhood. Lancet. 1997;350(9071):91-5.

36. Heymann S, Delaloge S, Rahal A, Caron O, Frebourg T, Barreau L, Pachet C, Mathieu MC, Marsiglia H, Bourgier C. Radio-induced malignancies afte breast cancer postoperative radiotherapy in patients with Li-Fraumeni syndrome. Radiat Oncol. 2010;5:104.

37. Salmon A, Amikam D, Sodha N, Davidson S, Basel-Vanagaite L, Eeles RA, Abeliovich D, Peretz T. Rapid development of post-radiotherapy sarcoma and breast cancer in a patient with a novel germline 'de-novo' TP53 mutation. Clin Oncol (R Coll Radiol). 2007:19(7):490-3.

\section{Publisher's Note}

Springer Nature remains neutral with regard to jurisdictional claims in published maps and institutional affiliations.

\section{Ready to submit your research? Choose BMC and benefit from}

- fast, convenient online submission

- thorough peer review by experienced researchers in your field

- rapid publication on acceptance

- support for research data, including large and complex data types

- gold Open Access which fosters wider collaboration and increased citations

- maximum visibility for your research: over $100 \mathrm{M}$ website views per year

At BMC, research is always in progress.

Learn more biomedcentral.com/submissions 